\title{
Terahertz surface plasmons in optically pumped graphene structures
}

\author{
A. A. Dubinov ${ }^{1,2}$, V. Ya. Aleshkin ${ }^{2}$, V. Mitin $^{3}$, T. Otsuji ${ }^{4,5}$, and V. Ryzhii ${ }^{1,5}$ \\ ${ }^{1}$ Computational Nanoelectronics Laboratory, University of Aizu, Aizu-Wakamatsu 965-8580, Japan \\ ${ }^{2}$ Institute for Physics of Microstructures, Russian Academy of Sciences, Nizhny Novgorod 603950, Russia \\ ${ }^{3}$ Department of Electrical Engineering, University at Buffalo, State University of New York, NY 14260, USA \\ ${ }^{4}$ Research Institute for Electrical Communication, Tohoku University, Sendai 980-8577, Japan \\ ${ }^{5}$ Japan Science and Technology Agency, CREST, Tokyo 107-0075, Japan
}

(Dated: March 17, 2021)

\begin{abstract}
We analyze the surface plasmons (SPs) propagating along the optically pumped single-graphene layer (SGL) and multiple-graphene layer (MGL) structures. It is shown that at sufficiently strong optical pumping when the real part of dynamic conductivity of SGL and MGL structures becomes negative in the terahertz $(\mathrm{THz})$ range of frequencies due to the interband population inversion, the damping of the $\mathrm{THz}$ SPs can give way to their amplification. This effect can be used in graphenebased $\mathrm{THz}$ lasers and other devices. Due to relatively small SP group velocity, the absolute value of their absorption coefficient (SP gain) can be large, substantially exceeding that of the optically pumped structures with the dielectric waveguide. The comparison of the SGL and MGL structures shows that to maximize the SP gain the number of GL layers should be properly choosen.
\end{abstract}

\section{INTRODUCTION}

Optical excitation of graphene can result in the interband population inversion [1, 2]. At sufficiently strong excitation, the interband emission of photons can prevail over the intraband (Drude) absorption. In this case, the real part of the dynamic conductivity Re $\sigma_{\omega}$ becomes negative. Due to the gapless energy spectrum of graphene [3], Re $\sigma_{\omega}$ can be negative at relatively low frequencies $\omega$, for instance, those in the terahertz $(\mathrm{THz})$ range. This effect can be used in graphene-based $\mathrm{THz}$ optically pumped lasers with the Fabri-Perot resonators and the resonators based on dielectric or slot-line waveguides [4 [6]. As was previously pointed out [1] and analyzed 7], apart from the lasing associated with the stimulated generation of electromagnetic modes, the stimulated generation of different plasmons (with their conversion into electromagnetic radiation) can also be of practical interest. The plasma excitations in graphene were considered, in particular, in Refs. [8-13]. In this paper, we study the propagation and amplification of surface plasmons (SPs) in graphene structures under nonequilibrium conditions when $\operatorname{Re} \sigma_{\omega}<0$ due to optical pumping. We consider a single-graphene layer (SGL) and a multiple-graphene layer (MGL) on a substrate. As demonstrated recently (see the review paper 14] and the references therein), the MGLs, which constitute stacks of disoriented (non-Bernal stacked) GLs, exhibit similar electron and optical properties as individual GLs, while the electron momentum relaxation time in such MGLs can be extremely long. The latter circumstance implies that the intraband absorption of photons and plasmons can be effectively diminished. This makes the MGLs very prospective for different optoelectronic devices considering that the net dynamic conductivity along GLs increases with increasing their number in the MGL structure. The MGL structures can be particularly effective in lasers in which the $\mathrm{THz}$ modes are supported by the external resonator or waveguide [5, 6], THz photodetectors 15 17], and transit-time $\mathrm{THz}$ oscillators [18, 19].

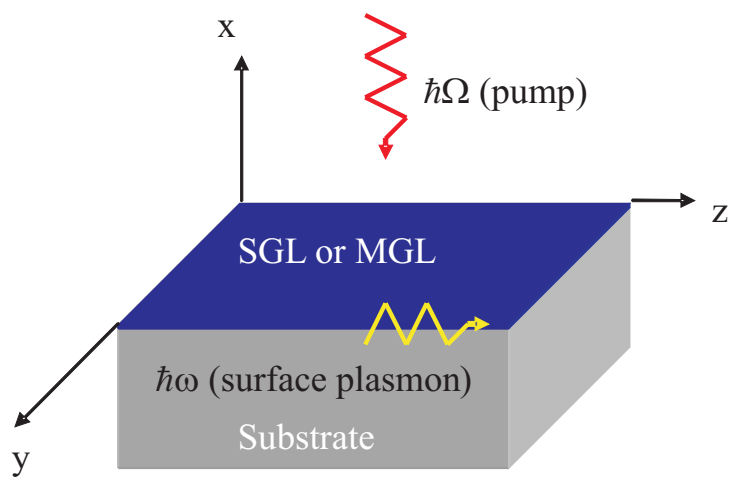

FIG. 1: Schematic view of the SGL/MGL structure under consideration.

However, SGL and MGL structures can play the dual role: the imaginary parts of their dynamic conductivity provides the mode localization near the SGL or MGL (i.e., the formation of SPs), while the real part provide absorption or amplification of plasmons. Due to this, the MGL structures as SP waveguides are not always superior over the SGL structures. Since the thickness, $d$, of the MGL structure with even relatively large number, $K$, of GLs is rather small in comparison with the wavelength of the plasmons under consideration, we shall consider the SGL and MGL structure alike. The real MGL usually include a highly conducting bottom GL (at the interface between the substrate and other GLs) with rather high electron density [14, 20]. Although this bottom GL can be in some way removed, we will take it into account.

It is assumed that the GL or MGL structure is illuminated from the top by light with the energy of photons $\hbar \Omega$. Under optical excitation, the electron and hole densities exceed substantially their equilibrium values. Due to this, one can consider the electron and hole systems under 

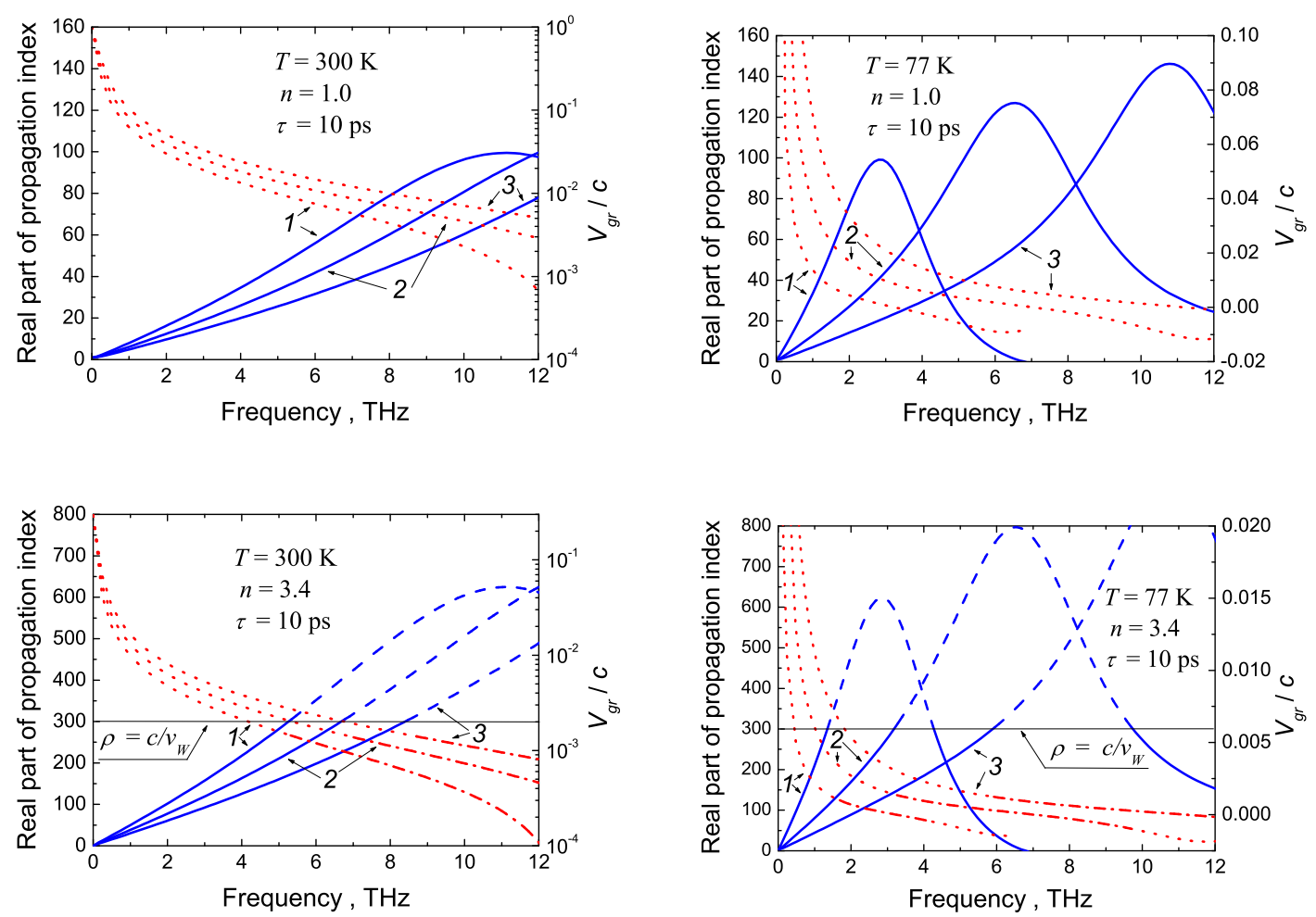

FIG. 2: Frequency dependence of the real part of propagation index Re $(\rho)$ (solid lines) and the SP group velocity normalized by the speed of light (dotted lines) in SGL structures at different temperatures $(T=300 \mathrm{~K}$ and $T=77 \mathrm{~K}$ ) and different quasi-Fermi energies $\left(1-\varepsilon_{F}^{T}=0 \mathrm{meV}, 2-\varepsilon_{F}^{T}=10 \mathrm{meV}\right.$, and $\left.3-\varepsilon_{F}^{T}=20 \mathrm{meV}\right)$ for SGL structures with different substrate refraction index $(n=1.0$ and $n=3.4)$. Dashed and dashed-doted lines in figures for $n=3.4$ correspond to the ranges where the effects of spatial dispersion might be essential.

consideration as characterized by the quasi-Fermi energies $\pm \varepsilon_{F}^{(k)}$, respectively, and the effective temperature $T$. A distinction in the Fermi energies in GLs with different indices is due to the attenuation of incident optical radiation associated with its absorption in the GLs closer to the structure top: $\varepsilon_{F}^{(k)}<\varepsilon_{F}^{(K)}=\varepsilon_{F}^{T}$. The electron-hole system in the bottom GL in MGLs is characterized by the unified Fermi energy $\varepsilon_{F}^{B}$ determined by the doping of this GL. If the characteristic time, $\tau_{0}$, of the emission of the optical phonon by an electron or a hole is much shorter than the time of pair collisions, the photoexcited electrons and holes emit a cascade of optical phonons and occupy low energy states in the conduction and valence bands, respectively. In this case, the contribution of optical excitation to the heating of the electron-hole system is small, so that the effective temperature $T$ is close to the lattice temperature $T_{l}$ [21]. In the opposite case, the photoexcited electrons and holes are thermalized, and their effective temperature is determined by the pumping photon energy $\hbar \Omega$ and the rate of electron and hole energy relaxation. In such a situation, the effective temperature can be elevated, so that somewhat stronger optical pumping might be needed to fullfil the condition $\operatorname{Re} \sigma_{\omega}<0$ [22].

\section{DYNAMIC CONDUCTIVITY OF SGL AND MGL STRUCTURES}

The net dynamic conductivity in the lateral direction of a structure with $K$ GLs at the signal frequency $\omega$ can be presented as the sum of the contributions of the individual GLs $\sigma_{\omega}^{(k)}(k=1,2, \ldots, K)$ and the bottom GL $\sigma_{\omega}^{B}$

$$
\sigma_{\omega}=\sum_{k=1}^{K} \sigma_{\omega}^{(k)}+\sigma_{\omega}^{B}
$$

Considering the expressions for $\sigma_{\omega}^{(k)}$ and $\sigma_{\omega}^{B}$ obtained previously (see, for instance, Refs, [10, 23] ), one can arrive at the following: 


$$
\begin{gathered}
\sigma_{\omega}^{(k)}=\left(\frac{e^{2}}{4 \hbar}\right)\left\{\frac{8 k_{B} T \tau}{\pi \hbar\left(1-i \omega^{2} \tau\right)} \ln \left[1+\exp \left(\frac{\varepsilon_{F}^{(k)}}{k_{B} T}\right)\right]+\tanh \left(\frac{\hbar \omega-2 \varepsilon_{F}^{(k)}}{4 k_{B} T}\right)-\frac{4 \hbar \omega}{i \pi} \int_{0}^{\infty} \frac{G\left(\varepsilon, \varepsilon_{F}^{k}\right)-G\left(\hbar \omega / 2, \varepsilon_{F}^{k}\right)}{(\hbar \omega)^{2}-4 \varepsilon^{2}} d \varepsilon\right\} \\
\sigma_{\omega}^{B}=\left(\frac{e^{2}}{4 \hbar}\right) \frac{4 k_{B} T \tau_{B}}{\pi \hbar\left(1-i \omega \tau_{B}\right)} \ln \left[1+\exp \left(\frac{\varepsilon_{F}^{B}}{k_{B} T}\right)\right] \\
+\left(\frac{e^{2}}{4 \hbar}\right)\left\{1-\left[1+\exp \left(\frac{\hbar \omega / 2-\varepsilon_{F}^{B}}{k_{B} T}\right)\right]^{-1}-\left[1+\exp \left(\frac{\hbar \omega / 2+\varepsilon_{F}^{B}}{k_{B} T}\right)\right]^{-1}-\frac{4 \hbar \omega}{i \pi} \int_{0}^{\infty} \frac{G\left(\varepsilon, \varepsilon_{F}^{B}\right)-G\left(\hbar \omega / 2, \varepsilon_{F}^{B}\right)}{(\hbar \omega)^{2}-4 \varepsilon^{2}} d \varepsilon\right\}
\end{gathered}
$$

Here, $e$ is the electron charge, $\tau_{B}$ and $\tau$ are the electron and hole momentum relaxation times in the bottom and other GLs, respectively, $\hbar$ is the reduced Planck constant, $k_{B}$ is the Boltzmann constant, and

$$
G\left(\varepsilon, \varepsilon^{\prime}\right)=\frac{\sinh \left(\varepsilon / k_{B} T\right)}{\cosh \left(\varepsilon / k_{B} T\right)+\cosh \left(\varepsilon^{\prime} / k_{B} T\right)} .
$$

In the case of SGL structure, one should put $K=1$ and $\sigma_{\omega}^{B}=0$. For the MGL structures without the bottom GL, one can use Eq. (3) formally setting $\sigma_{\omega}^{B}=0$.

The quasi-Fermi energies in the GLs with $k \geq 1, \varepsilon_{F}^{(k)}$, are mainly determined by the electron (hole) density in this layer $\Sigma_{k}$, with $\varepsilon_{F}^{(k)} \propto \sqrt{\Sigma^{(k)}}$ (at sufficiently strong degeneracy of the electron and hole systems), i.e., by the rate of photogeneration $G_{\Omega}^{(k)}$ by the optical radiation at the $k$-th GL plane. Considering the attenuation of the optical pumping radiation due to its absorption in each GL, one can obtain that $\varepsilon_{F}^{(k)}$ can be expressed via the quasi-Fermi energy in the topmost GL $\varepsilon_{F}^{T}=\varepsilon_{F}^{(K)}$, which, in turn, is a function of the the intensity of incident pumping radiation $I_{\Omega}$. Considering the MGL structures, the $\varepsilon_{F}^{(k)}$ versus $\varepsilon_{F}^{T}$ dependence was found as previously [6].

\section{DISPERSION EQUATION FOR SP.}

Using the the Maxwell equations, in which the current associated with electron-hole system is given by of

$$
j_{x}=0, \quad j_{y}=0, \quad j_{z}=\sigma_{\omega} E_{z} \delta(x),
$$

The components of the electric and magnetic fields, $\mathbf{E}=$ $\left(E_{x}, 0, E_{z}\right)$ and $\mathbf{H}=\left(0, H_{y}, 0\right)$ can be presented in the following form:

$$
E_{x} \propto E_{z} \propto H_{y} \propto \exp \left(i \frac{\omega}{c} \sqrt{1-\rho^{2}} x+i \frac{\omega}{c} \rho z-i \omega t\right),
$$

at $x \geq 0$, and

$$
E_{x} \propto E_{z} \propto H_{y} \propto \exp \left(-i \frac{\omega}{c} \sqrt{n^{2}-\rho^{2}} x+i \frac{\omega}{c} \rho z-i \omega t\right)
$$

at $x<0$. Here, $\omega$ is the signal (plasmon) frequency, $c$ is the speed of light, $n$ is the refraction index (generally complex) of the substrate, $\rho$ is the complex propagation index (which relates to the wavenumber $q_{z}=\rho \omega / c$ ), the axis $x$ is directed perpendicular to the structure plane, the axis $z$ corresponds to the in-plane direction along the SP propagation, and $\delta(x)$ is the Dirac delta function. The latter describes the localization of the plasmon near the SGL or MGL structure plane. Considering Eqs. (6) and (7), from the Maxwell equations we obtain the following dispersion equation for SPs:

$$
\sqrt{n^{2}-\rho^{2}}+n^{2} \sqrt{1-\rho^{2}}+\frac{4 \pi}{c} \sigma_{\omega} \sqrt{1-\rho^{2}} \sqrt{n^{2}-\rho^{2}}=0 .
$$

Equation (8) can be derived from that obtained previously using a different approach [12, 13]. What is important for the present consideration is that $\sigma_{\omega}$ is determined by both nonequilibrium electron and hole components with, in particular, $\operatorname{Re}\left(\sigma_{\omega}\right)<0$. Solving Eq. (8) with respect to $\rho$, one can find the propagation index Re $(\rho)$ and the SP absorption (amplification) coefficient

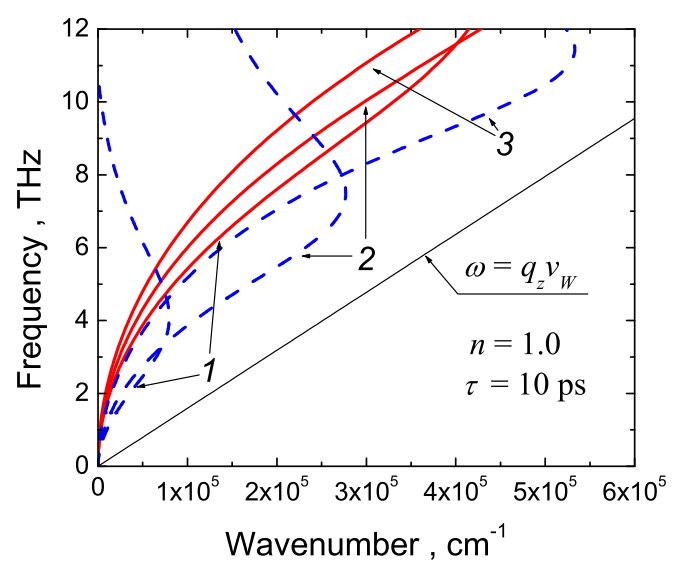

FIG. 3: Dispersion of SPs in a SGL structure at $T=300 \mathrm{~K}$ (solid lines) and $T=77 \mathrm{~K}$ (dashed lines) with the same as in Fig. 2 values of the quasi-Fermi energy. 

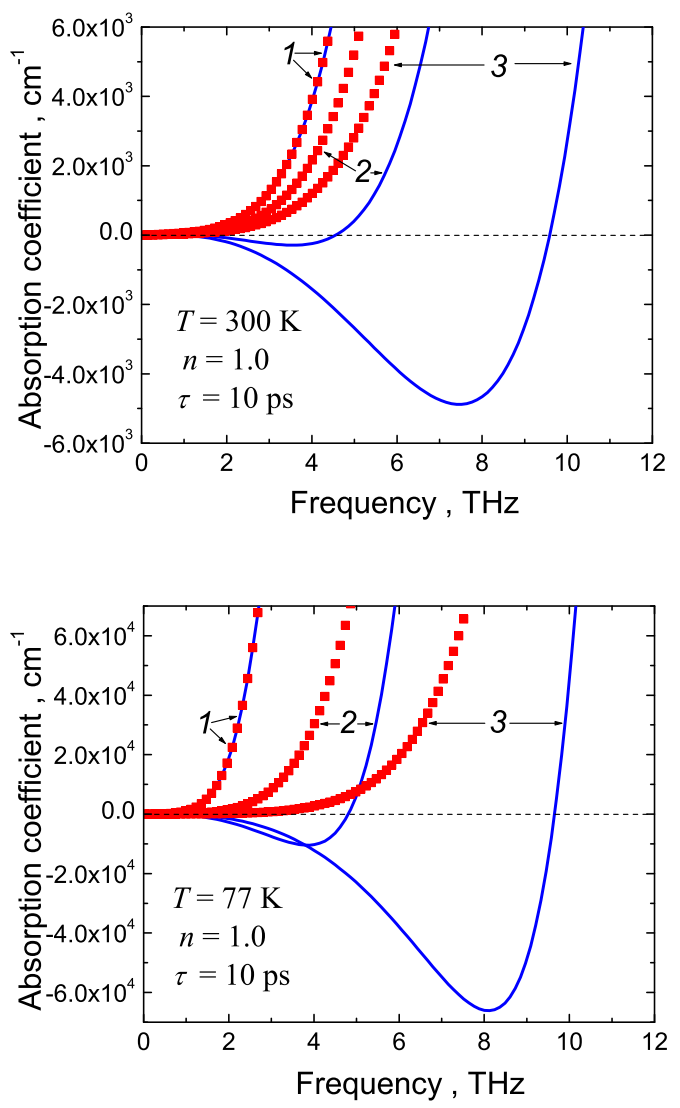

FIG. 4: Frequency dependences of SP absorption coefficient $2 \operatorname{Im}(q)$ in a SGL structure at $T=300 \mathrm{~K}$ (upper panel) and $T=77 \mathrm{~K}$ (lowe panel) and different quasi-Fermi energies (1 $\varepsilon_{F}^{T}=0 \mathrm{meV}, 2-\varepsilon_{F}^{T}=10 \mathrm{meV}$, and $\left.3-\varepsilon_{F}^{T}=20 \mathrm{meV}\right)$. Markers show the dependences for equilibrium electron-hole systems calculated using the derived formulas as well as using 12] for intrinsic and doped SGL structures.

$2 \operatorname{Im}(q)=2 \operatorname{Im}(\rho \omega / c)$ or the SP gain $-2 \operatorname{Im}(q)$. In the cases when $n=1$ (SGL or MGL structure levitating in the free space), Eq. (8) obviously yields

$$
\rho=\sqrt{1-\frac{c^{2}}{4 \pi^{2} \sigma_{\omega}^{2}}} .
$$

\section{RESULTS OF CALCULATIONS}

\section{A. SP spectra and SP damping/amplification}

Figure 2 presents the frequency dependences of the real part of the propagation index $\operatorname{Re}(\rho) \propto \operatorname{Re}(q)$ shown by solid lines calculated for SGL structures with different substrate refraction indices $n$ at different temperatures $T$ and different values of the quasi-Fermi energies $\varepsilon_{F}^{T}$ (i.e., for different pumping intensities). Dotted lines in Fig. 2 correspond to the frequency dependences of the group velocity of SPs normalized by the speed of light in vac-
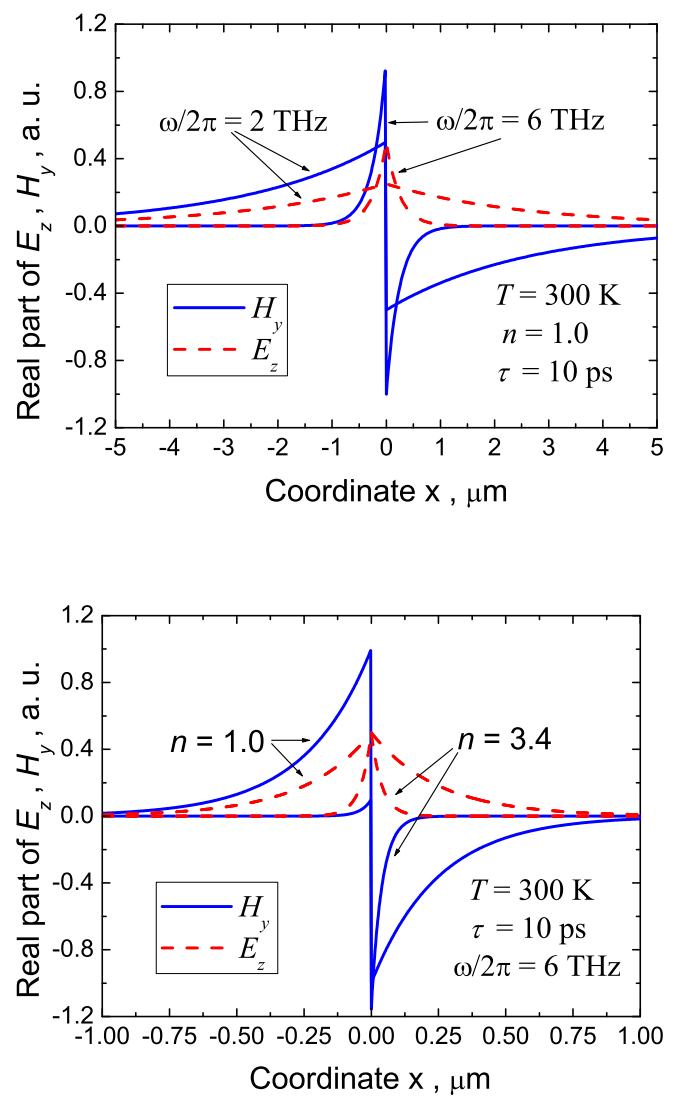

FIG. 5: Spatial distributions of the real parts of electric and magnetic fields in SGL structures at different frequencies (upper panel) and different substrate refraction indices (lower panel): $T=300 \mathrm{~K} \cdot \varepsilon_{F}^{T}=20 \mathrm{meV}$, and $\tau=10 \mathrm{ps}$.

uum $c$. In our model we disregarded the effect of spatial dispersion on the dynamic conductivity of SGL and MGL structures, i.e., the dependence of $\sigma_{\omega}$ on the SP wavenumber $q_{z}$ in Eqs. (1) - (4). This is valid if $\rho<c / v_{W}$ or $\omega<\operatorname{Re}\left(q_{z}\right) v_{W}$ [10], where $v_{W}=10^{8} \mathrm{~cm} / \mathrm{s}$ is the characteristic velocity of electrons in holes in graphene. In the case of SGL structures with $n=1$, these inequalities are satisfied in the frequency range under consideration (see Figs. 2 and 3). However, as follows from Fig. 2 at larger $n$, the validity of the dependences obtained is limited by relatively low frequencies $(\omega / 2 \pi \lesssim 5-8 \mathrm{THz}$ for $n=3.4$ at $T=300 \mathrm{~K})$. One can see from Fig. 2 that the SP group velocity can be negative (at $T=77 \mathrm{~K}$ ) at elevated frequencies. This corresponds to backward waves [24, 25] and occurs at the frequencies at which the imaginary part of the dynamic conductivity is determened primarily by the interband transitions. Figure 3 shows the spectra of SPs, i.e., $\omega$ versus $\operatorname{Re}\left(q_{z}\right)$ dependences in the SGL structures at different temperatures at the same pumping conditions as in Fig. 2.

Figure 4 shows the SP absorption coefficient $2 \operatorname{Im}\left(q_{z}\right)$ as a function of frequency calculated for different tem- 


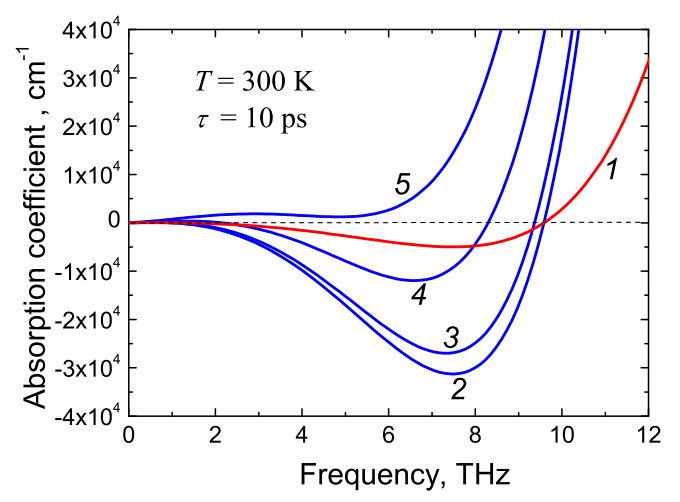

FIG. 6: Frequency dependences of SP absorption for SGL structures with different substrate refraction indices $n: 1$ $n=1.0,2-n=3.4,3-n=3.4+i 0.01$, and $4-n=3.4+i 0.05$, and $5-n=3.4+i 0.1\left(T=300 \mathrm{~K}, \varepsilon_{F}^{T}=20 \mathrm{meV}\right)$.

peratures $T$ and different values of the quasi-Fermi energies $\varepsilon_{F}^{T}$. One can see that an increase in $\varepsilon_{F}^{T}$ leads to widening of the frequency range where $\operatorname{Im}\left(q_{z}\right)<0$ and a marked increase in the absolute value of the absorption coefficient in this range. The dependences indicated by markers were calculated for equilibrium SGLs using the above formulas which in the absence of optical pumping at $n=1$ coincide with those obtained previously [12]. The markers correspond to intrinsic (with the Fermi energy $\left.\varepsilon_{F}=0\right)$ and doped SGL structures. The dependences for doped GL structures at the equilibrium correspond to the Fermi energies $\varepsilon_{F}$ in doped SGLs at the equilibrium equal to the quasi-Fermi energies $\varepsilon_{F}^{T}$ in the optically pumped SGLs. Clear distinctions in these dependences in the equilibrium and pumped GLs are associated with the contributions of both electrons and holes to the negative dynamic conductivity due to the interband transitions (in the latter).

Figure 5 shows the spatial distributions (in the direction perpendicular to the SGL plane) of the SP electric and magnetic fields in the SGL structures with different substrate refraction indices.

\section{B. Role of substrate and intraband absorption}

Figure 6 shows the frequency dependences of the SP absorption coefficient calculated for SGLs with the substrates with different real and imaginary parts of the refraction index (different absorption in the substrate). As follows from Fig. 6, the contribution of the substrate to the SP absorption can be insignificant at the realistic values of the imaginary part of the substrate refraction index. In particular, in the case of the substrate made of undoped $\operatorname{Si}\left(\operatorname{Im}(n) \simeq 3 \times 10^{-4}[26]\right)$, the imaginary part of the refraction index can be smaller than those used in the calculations of curves 3- 5 in Fig. 6.

Figure 7 shows the frequency dependences of the real part of the propagation index and the SP absorption co-
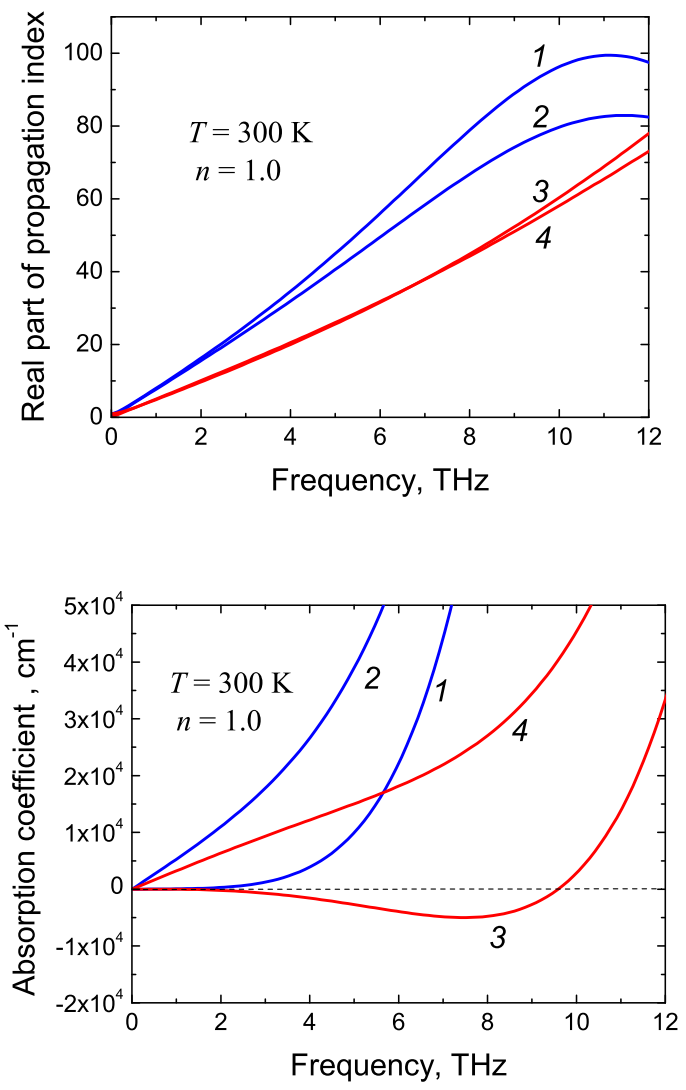

FIG. 7: Real part of SP propagation index (upper panel) and absorption coefficient (lower panel) in SGL structures as functions of frequency: $1-\varepsilon_{F}^{T}=0 \mathrm{meV}, \tau=10 \mathrm{ps}$, $2-\varepsilon_{F}^{T}=0 \mathrm{meV}, \tau=0.1 \mathrm{ps}, 3-\varepsilon_{F}^{T}=20 \mathrm{meV}, \tau=10 \mathrm{ps}$, and $4-\varepsilon_{F}^{T}=20 \mathrm{meV}, \tau=0.1 \mathrm{ps}$.

efficient. These dependences were calculated for SGL structures with different electron and hole momentum relaxation times $(\tau=10 \mathrm{ps}$ and $\tau=0.1 \mathrm{ps})$ for different $\varepsilon_{F}^{T}$, i.e., for different pumping intensities assuming that $T=300 \mathrm{~K}$. As seen from Fig. 7, in the SGL structure with a relatively long momentum relaxation time $(\tau=10 \mathrm{ps})$, the SP absorption coefficient changes its sign at moderate values of $\varepsilon_{F}^{T}\left(\varepsilon_{F}^{T} \lesssim 20 \mathrm{meV}\right)$. However when $\tau=0.1 \mathrm{ps}$, the SP absorption coefficient does not change the sign at least at $\varepsilon_{F}^{T} \sim 20 \mathrm{meV}$ (see below), although it is markedly smaller than in the equilibrium conditions (without pumping). However, at elevated pumping intensities (elevated values of the quasi-Fermi energy) the absorption coefficient in the SLG structures even with short momentum relaxation times can become negative although at higher frequencies (see Fig. 8).

\section{Comparison of SGLs and MGLs}

In Figs. 9 and 10, we compare the real parts of the SP propagation index, absorption coefficients, the SP group velocities in the optically pumped SGLs and MGLs 


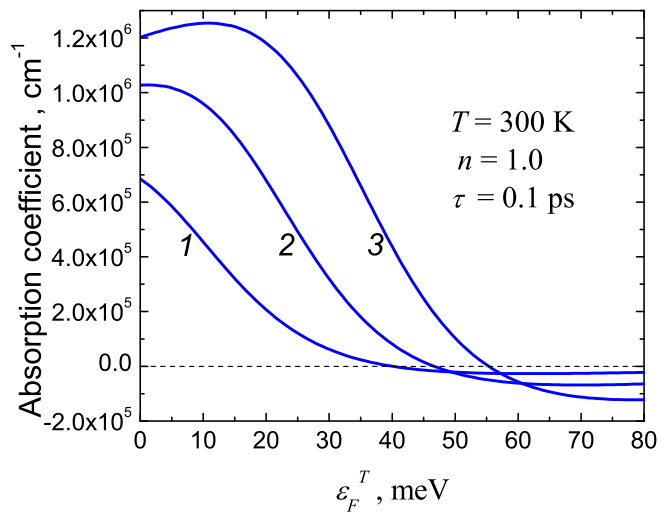

FIG. 8: SP absorption coefficient as a function of the quasiFermi energy in SGL structures with short momentum relaxation time $(\tau=0.1 \mathrm{ps})$ at $1-\omega / 2 \pi=15 \mathrm{THz}, 2-\omega / 2 \pi=$ $20 \mathrm{THz}, 3-\omega / 2 \pi=25 \mathrm{THz}$.

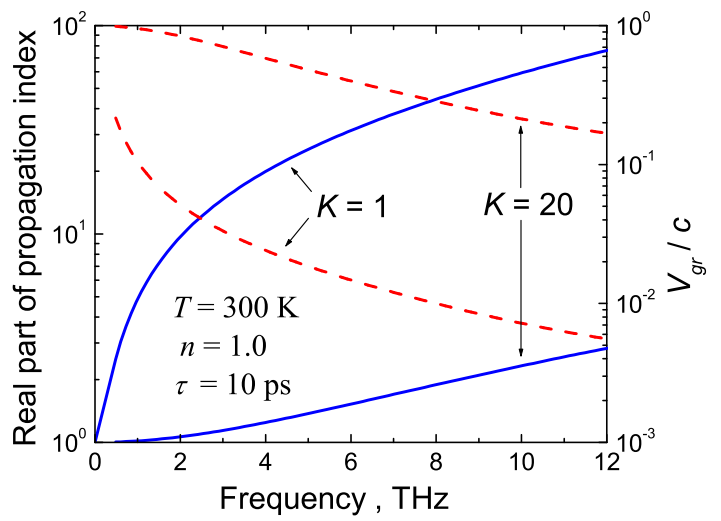

FIG. 9: Frequency dependences of real part of SP propagation index (solid lines) and normalized group velocity (dashed lines) for SGL structure $(K=1)$ and MGL structure $(K=20)$ at $\varepsilon_{F}^{T}=20 \mathrm{meV}$.

$\left(\varepsilon_{F}^{T}=20 \mathrm{meV}\right)$. As seen, the SP characteristics in SGLs and MGLs are markedly different. In particular, SGLs exhibit substantially stronger (about 50 times in the peaks) amplification (higher plasmon gain) in a wide frequencies range (from 4 to $8 \mathrm{THz}$ ) at $\tau=10 \mathrm{ps}$. This is attributed to markedly different values of the SP group velocities in SGLs (relatively small plasmon group velocity) and in MGLs (in which this velocity is relatively high), as shown in Fig. 9, which, in turn, is due to different net electron and hole densities and different widths of the SP electric field localization that is clearly seen in Fig. 11. An example of the dependence of the SP absorption coefficient in the structures with optically pumped GLs on the number of the latter is demonstrated in Fig. 12. Thus, at the same values of the optical pumping intensity and the electron and hole momentum relaxation time, SPs in SGL structures can exhibit stronger

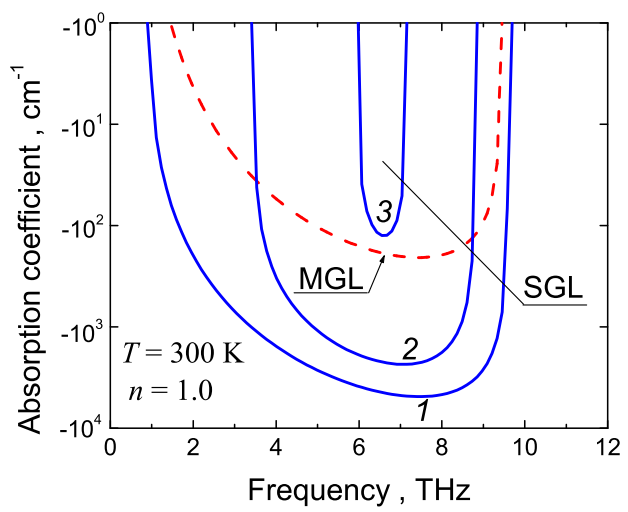

FIG. 10: Frequency dependences of SP absorption coefficient at $\varepsilon_{F}^{T}=20 \mathrm{meV}$ for SGL structure with $1-\tau=10 \mathrm{ps}, 2-$ $\tau=1.0 \mathrm{ps}$, and 3- $\tau=0.54 \mathrm{ps}$ and for MGL structure with $K=20$ (dashed line) and $\tau=10 \mathrm{ps}$.

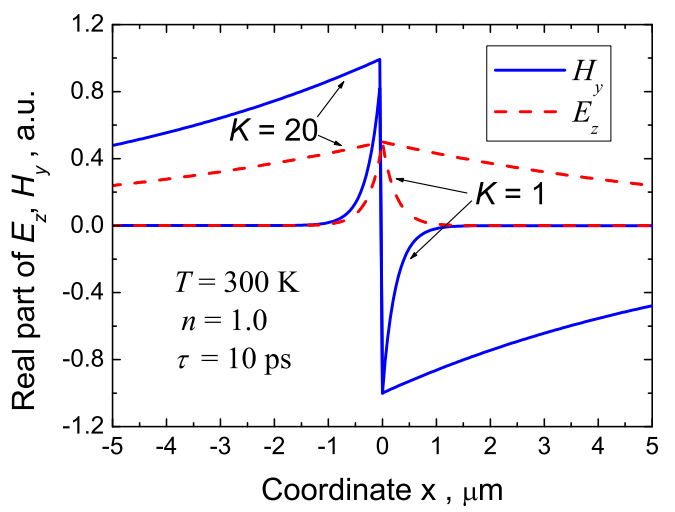

FIG. 11: Spatial distribution of real parts of the SP electric and magnetic fields in a SGL structure and MGL structures with $K=20$ at $T=300 \mathrm{~K}, \varepsilon_{F}^{T}=20 \mathrm{meV}, \tau=10 \mathrm{ps}$, and $\omega / 2 \pi=6 \mathrm{THz}$.

amplification than MGL structures. However, as demonstrated [14], the momentum relaxation time in epitaxially grown MGLs can be rather long, so MGL structures might be preferable in applications. Taking into account the real possiblity of a large difference in the electron and hole relaxation times in SGL and MGL structures, in Fig. 10, we demonstrate that the SP amplification in the MGL structure with $K=20$ and $\tau=10$ ps can exceed that in the SGL structure with smaller $\tau$. Hence, to achieve the SP maximum gain, the number of GL layers should be optimal.

A decrease in the SP gain with increasing $K$ is in contrast to the behavior the electromagnetic modes in optically pumped MGL structures with the dielectric waveguide considered by us previously [6]. For comparison, the pertinent dependence taken from Ref. [6] for the waveguide thickness $L=5 \mu \mathrm{m}$ (at the same quality of GLs and 


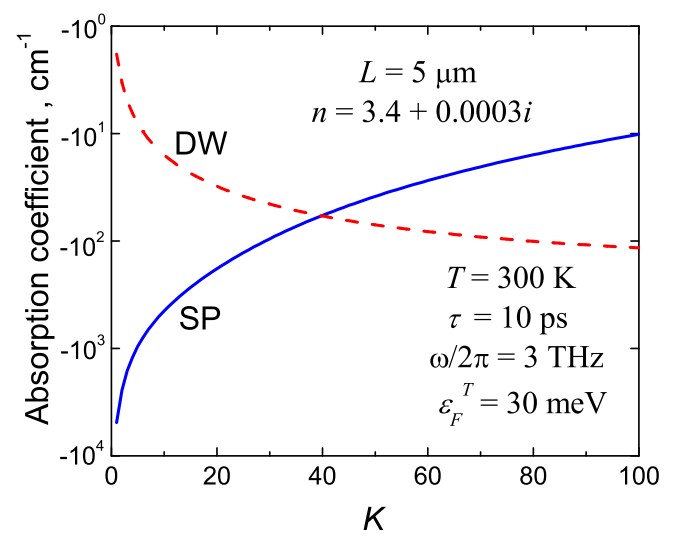

FIG. 12: Absorption coefficients of SPs (solid line) and an electromagnetic mode (dashed line) in a dielectric waveguide (DW) [6] as functions of the number of GLs $K$.

pumping conditions) is shown in Fig. 12 as well.

\section{CONCLUSIONS}

We studied the SPs in optically pumped SGL and MGL structures. Using the developed model, we calculated the SP dispersion relations, spatial distributions of their electric and magnetic fields, and frequency dependences of the absorption coefficient as functions of the optical pumping and factors determining the intraband absorp- tion in GLs and the substrate. It was demonstrated that at sufficiently strong but realistic optical pumping, the SP absorption coefficient can be negative (so that the gain is positive) in the certain range of frequencies. The absolute value of the SP absorption coefficient (plasmon gain) in SGL structure can be fairly large and markedly exceed the gain of electromagnetic modes in the dielectric waveguides with optically pumped SGL or MGL structures. In contrast to the cases of amplification of electromagnetic modes in the structures with optically pumped SGL and MGL and dielectric waveguide, the SP amplification weakens with increasing number of GLs $K$. However, due to possibility of rather long momentum relaxation times of electrons and holes in MGL structures, the SP maximum gain can be achieved at optimal values of the nimber of GLs. The effect of SP amplification in optically pumped SGL and MGL structures can be used in $\mathrm{THz}$ lasers (with the conversion of SPs to the output electromagnetic modes). The variation of the SP characteristics by optical pumping might be useful in different GL-based plasma-wave devices.

\section{Acknowledgments}

The authors are grateful to M. Ryzhii for comments. This work was supported by the Japan Science and Technology Agency, CREST, Japan and partially by the Federal Russian Program "Scientific and Educational Staff", Russia.
[1] V. Ryzhii, M. Ryzhii, and T. Otsuji, J. Appl. Phys. 101, 083114 (2007).

[2] A. Satou, F. T. Vasko, and V. Ryzhii, Phys. Rev. B 78, 115431 (2008).

[3] A. H. Castro Neto, F. Guinea, N. M. R. Peres, K. S. Novoselov, and A. K. Geim, Rev. Mod. Phys. 81, 109 (2009).

[4] A. A. Dubinov, V. Ya. Aleshkin, M. Ryzhii, T. Otsuji, and V. Ryzhii, Appl. Phys. Exp. 2, (2009).

[5] V.Ryzhii, M. Ryzhii, A. Satou, T. Otsuji, A. A. Dubinov, and V. Ya. Aleshkin, J. Appl. Phys., 106, 084507 (2009).

[6] V. Ryzhii, A. A. Dubinov, T. Otsuji, V. Mitin, and M. S. Shur J. Appl. Phys. 107, 054505 (2010).

[7] F. Rana, IEEE Trans. Nanotechnol. 7, 91 (2008).

[8] V. Ryzhii, Jpn. J. Appl. Phys. (Part 2) 45, L923 (2006).

[9] O. Vafek, Phys. Rev. Lett., 97, 266406 (2006).

[10] L. A. Falkovsky and A. A. Varlamov, Eur. Phys. J. B 56, 281 (2007).

[11] V. Ryzhii, A. Satou, and T. Otsuji, J. Appl. Phys. 101, 024509 (2007).

[12] G. W. Hanson, J. Appl. Phys. 103, 064302 (2008).

[13] M. Jablan, H. Buljan, and M. Solijacic, Phys. Rev. B 80, 245435 (2009).

[14] M. Orlita and M. Potemski, Semicond. Sci. Technol. 25, 063001 (2010).
[15] F. T. Vasko and V. Ryzhii, Phys. Rev B 77, 195433 (2008).

[16] F. Xia, T. Murller, Y.-M. Lin, A. Valdes-Garsia, and F. Avouris, Nat. Nanotechnol. 4, 839 (2009).

[17] V. Ryzhii, M. Ryzhii, V. Mitin, and T. Otsuji, J. Appl. Phys. 107, 054512 (2010).

[18] V. Ryzhii, M. Ryzhii, V. Mitin, and M. S. Shur, Appl. Phys. Express 2, 034503 (2009).

[19] M. Ryzhii, V. Ryzhii, T. Otsuji, V. Mitin, and M. S. Shur, Phys. Rev. B 82, 075419 (2010).

[20] F. Varchon, R. Feng, J. Hass, X. Li, B. Ngoc Nguyen, C. Naud, P. Mallet, J.-Y. Veuillen, C. Berger, E. H. Conrad, and L. Magaud, Phys. Rev. Lett. 99, 126805 (2007).

[21] V.Ryzhii, M.Ryzhii, and T.Otsuji. Phys. Stat. Sol. (c) 5, 261 (2008).

[22] A.Satou, T. Otsuji, and V. Ryzhii, Abst. of the 2nd Int. Symp. on Graphene Devices: Technology, Physics, and Modeling (ISGD2010), Sendai, Japan Oct.27-29, 2010.

[23] L. A. Falkovsky and S. S. Pershoguba, Phys. Rev. B 103, 064302 (2008).

[24] A. A. Oliner and T. Tamir, J. Appl. Phys. 33, 231 (1962).

[25] L. Novotny and C. Hafner, Phys. Rev. E, 50, 4094 (1994).

[26] E. D. Palik Handbook of Optical Constants of Solids (Academic Press New York, 1998). 